\title{
Impact of Systemic Therapy and Recurrence Pattern on Survival Outcome after Radiofrequency Ablation for Colorectal Liver Metastases
}

\author{
Axel Stang ${ }^{1 凶}$, Marcello Donati², Hauke Weilert³ ${ }^{3}$ Karl Jürgen Oldhafer ${ }^{4}$ \\ 1. Department of Hematology, Oncology, \& Palliative Care, Asklepios Hospital Barmbek, Semmelweis University of Medicine, Asklepios Campus Hamburg, Germany. \\ 2. Department of Surgery \& Medical-Surgical Specialities, General \& Oncologic Surgery Unit, Vittorio-Emanuele University Hospital, University of Catania, Italy. \\ 3. Department of Hematology \& Oncology, Asklepios Hospital Altona, Semmelweis University of Medicine, Asklepios Campus Hamburg, Germany; \\ 4. Department of General \& Abdominal Surgery, Asklepios Hospital Barmbek, Semmelweis University of Medicine, Asklepios Campus, Germany. \\ $\triangle$ Corresponding author: Axel Stang, MD, Department of Hematology, Oncology, and Palliative Care, Asklepios Hospital Barmbek, Semmelweis University of \\ Medicine, Asklepios Campus Hamburg, Rübenkamp 220, Hamburg 22291, Germany. Telephone number: +49-40-181882-3831, \\ Fax number: +49-40-181882-3309, Email: a.stang@asklepios.com.
}

(C) Ivyspring International Publisher. Reproduction is permitted for personal, noncommercial use, provided that the article is in whole, unmodified, and properly cited. See http://ivyspring.com/terms for terms and conditions.

Received: 2016.03.26; Accepted: 2016.07.09; Published: 2016.09.27

\begin{abstract}
Background: Most patients undergoing radiofrequency ablation (RFA) of colorectal liver metastasases (CLM) develop disease recurrence, but little is known about the effect of recurrence patterns and/or systemic therapy on outcome. In this study, we examined the recurrence patterns and survival after systemic therapy plus RFA in patients with unresectable CLM without extrahepatic disease. The aims were to analyze the effect of recurrence patterns on survival and to assess the relative benefit contributed by systemic therapy and local ablation to disease control and patient outcome.

Methods: From January 2002 to December 2012, 113 patients underwent RFA of liver-limited CLM after systemic therapy. Univariate and multivariate analyses for associations between clinical and/or treatment-related variables, recurrence-free survival (RFS), recurrence patterns, and overall survival (OS) were carried out.

Results: Of 113 patients, 105 (92.8\%) had disease recurrence (median RFS: 6.1 months). Lower post-recurrence OS was observed after early ( $\leq 6$ months) than after late recurrence ( 8.5 versus 24.0 months, $\mathrm{p}<0.001)$. Recurrence sites were RFA-sites only $(4.8 \%)$, liver-only $(57.1 \%)$, lung-only $(10.5 \%)$, or multiple (27.6\%); the corresponding post-recurrence OS was $21,19,39$, and 7 months $(p<0.001)$, respectively. Response to pre-RFA systemic therapy was the strongest predictor for OS (hazard ratio [HR] 5.28), RFS (HR 3.30), early (odds ratio [OR] 6.34) and multiple-site recurrence (OR 3.83) $(p<$ $0.01)$, respectively; only responders achieved 5 -year OS and RFS $(29 \%$ and $12 \%$ versus $0 \%$ and $0 \%$ for non-responders, $\mathrm{p}<0.001$, respectively).

Conclusions: Survival after RFA for liver-limited CLM is strongly linked to the timing and pattern of non-local disease recurrence. Local ablation efficacy is necessary but not sufficient to obtain long-term disease control. Effective pre-RFA systemic therapy does favourably affect the incidence, timing and patterns of recurrence and long-term survival and appears essential for the tailoring of RFA application to maximize patient benefit.
\end{abstract}

Key words: Colorectal cancer, metastases, radiofrequency ablation, recurrence, survival.

\section{Introduction}

The treatment options for patients with unresectable colorectal liver metastases (CLM) have evolved markedly, especially with the modern-agent systemic and local ablative therapies now available for routine use [1,2]. Among ablative therapies, radiofrequency ablation (RFA) is the most widely used for complete eradication of unresectable CLM.[3] Local recurrence (LR) rates after RFA of small $(\leq 3-4$ $\mathrm{cm})$ CLM in current studies are low $(<10 \%)$ [4], and reported 5-year overall survival (OS) rates following 
RFA for liver-limited CLM range between 17 to 51\% [5-9]. However, the standard treatment for unresectable CLM is systemic therapy, and the precise place of RFA within the treatment algorithm is not defined.

Treatment of liver-only CLM evolves towards combining local and systemic therapy, owing to the fact that it represents a systemic disease. The randomized phase II CLOCC trial supports this concept and documents improved 5-year OS from adding RFA upfront to chemotherapy in patients with up to 9 liver-limited CLM $[10,11]$. However, $>80 \%$ of patients' experience disease recurrence distant from RFA-treated CLM within 12 months, and virtually no RFA-treated patient achieves 5-year recurrence-free survival (RFS) [12]. Therefore, it is reasonable to consider that the recurrence pattern may play a role for survival outcome. However, while some data on recurrence patterns after RFA of liver-limited CLM exist $[13,14]$, little is known about the effect of these recurrence patterns on survival outcome.

With the availability of effective antineoplastic agents, response to modern-agent regimens has become an important prognostic factor and has been shown to convert unresectable CLM to respectability $[15,16]$. This raises the increasing need to define the results and place of RFA of CLM with regard to the responsiveness of disease to systemic therapy. Based on prior data suggesting a potential for long-term RFS after RFA of liver-limited CLM responding to chemotherapy $[17,18]$, we considered that the more common inverse phenomenon of disease recurrence was worthy of further study. An analysis of the relationship between response to pre-RFA systemic therapy, post-RFA recurrence patterns, and survival outcome may help to better define the current place of RFA in the multimodality treatment of unresectable liver-limited CLM and also aid to improve multimodal concepts and/or patient stratification in future clinical trials. Therefore, such analysis may be of interest for both clinical and research settings.

In the present study, we evaluated the incidence, timing, and patterns of recurrence and survival after systemic therapy plus RFA in patients with unresectable CLM without extrahepatic disease (EHD). The aims were to assess the effect of recurrence pattern on survival and to assess the relative benefit contributed by systemic therapy and local ablation to disease control and patient outcome.

\section{Materials and methods}

\section{Patient selection}

We performed a retrospective review of a prospectively collected database of patients who had received RFA of liver-limited CLM after systemic therapy at the Asklepios Hospital Barmbek between January 2002 and December 2012. The study was approved by the Ethics Committee the Asklepios Hospital Barmbek and carried out in accordance with the Declaration of Helsinki. All patients included into the database provided written informed consent for scientific evaluation of the data. Inclusion criteria for RFA treatment were: $\leq 5$ unresectable liver-only CLM of $\leq 5 \mathrm{~cm}$ maximum size, and anticipated life expectancy of $\geq 6$ months. Non-resectability was determined by two hepatobiliary surgeons and defined as technically impossible to achieve a margin-negative resection with preservation of $\geq 30 \%$ liver parenchyma (i.e. a margin-negative resection requires resection of 3 hepatic veins or both portal veins; or, a resection leaves $<2$ adequately perfused and drained segments). Inoperability was determined by two surgeons, one oncologist, and one anaesthetist and defined as contraindications to general anaesthesia (deterioration of general condition and/or cardio respiratory disease), inadequate liver function, Karnofsky performance score of less than $70 \%$, or patient's refusal. Exclusion criteria for RFA were proximity of CLM to major biliary structures or bleeding disorders.

\section{Pre-RFA assessments}

Assessments included performance status evaluation, liver function tests, carcinoembryonic antigen (CEA) evaluation, and contrast-enhanced computed tomography (CT) of the chest, abdomen, and pelvis. Response to systemic therapy was classified as partial response (PR), stable disease (SD), or progressive disease (PD) using the Cancer Response Evaluation Criteria In Solid Tumours (RECIST) [19].

\section{RFA procedure}

RFA was performed percutaneously under conscious sedation and analgesia and guided by ultrasonography (US) or CT. A 15-gauge multi-tined expandable RF needle electrode (LeVeen, Boston Scientific, Natick; 3 or $4 \mathrm{~cm}$ in diameter) was used connected to an RF generator (RF 3000, Boston Scientific, Natick, MA, USA). After stepwise increase of power, primary end points for a technically successful ablation were $\geq 2$ increases in tissue impedance. Routinely, contrast-enhanced US (SonoVue, Bracco, Milan, Italy) guided additional ablations if the ablation volume achieved was insufficient compared to the pre-RFA tumour size and/or margin. An RFA procedure was considered to be complete when the ablated area, as determined by the absence of contrast enhancement at US, 
encompassed the RFA-treated CLM by including a $\geq 0.5 \mathrm{~cm}$ ablative margin.

\section{Post-RFA assessments}

Assessments included contrast-enhanced CT of the chest, abdomen, and pelvis and CEA evaluation. Initial post-RFA CT imaging was performed 4 weeks after RFA treatment to establish a new baseline, thereafter every 3 months for the first 2 years, and then 6 monthly. Each follow-up study was compared to the contrast-enhanced CT images before RFA and the new baseline CT studies after RFA. Treatment efficacy was determined on according to the criteria proposed by the International Working Group on Image-Guided Tumour Ablation [3]. Local recurrence was defined as the development of new focal areas of contrast enhancement at follow-up CTs, either within or adjacent to the edge of RFA-treated CLM that were previously considered to be completely ablated. All other new contrast-enhancing focal lesions at other intra-/ and extrahepatic sites were considered new metastases and defined as intra- and/or extrahepatic recurrence. Procedural complications were determined according to the Society of Interventional Radiology (SIR) classification system [20]. Major complications were defined as events associated with substantial morbidity and disability, requiring surgical or radiological interventions, blood transfusion, significant medical therapies or longer hospital stay. All other complications were considered minor.

\section{Statistical analysis}

Quantitative and qualitative variables were summarized in terms of median (range), mean (standard deviation), and frequency (percentage). Comparisons between groups were analyzed with the chi-square or Fisher exact test for categorical variables and the Mann-Whitney $U$ test for continuous variables, as appropriate. Survival curves for OS and RFS were calculated using the Kaplan-Meier method and compared using log-rank tests. All variables associated with survival or recurrence patterns with $p$ $\leq 0.05$ in the univariate analyses were subsequently entered into a Cox multivariate proportional hazard model to assess their independent influence. Estimated hazards ratios (HRs) for survival duration and odds ratios (ORs) for predicting recurrence were ranked to assess the most important factor among independent factors identified on multivariate analysis. For all tests, p-values $\leq 0.05$ were considered statistically significant. Statistical analyses were performed using the SAS Statistical Software Version 9.1 (SAS, Institute Inc.; Cary, North Carolina, USA).

\section{Results}

\section{Patient characteristics}

Table 1 shows the baseline characteristics of the study population, which included a total of 113 patients (69 men, 44 women, median age: 70 years) with 279 CLM. The median number of RFA-treated CLM per patient was 2.5 (range 1-5), with a median size of the largest RFA-treated CLM of $2.8 \mathrm{~cm}$ (range: 0.6 to $5.0 \mathrm{~cm}$ ). With respect to the total exposure to antineoplastic agents, patients had received on average 3 (range 1-5) lines of systemic therapies before and after RFA: a total of 106 patients $(94 \%)$ had been treated with FOLFOX, 107 (95\%) with FOLFIRI, 63 (55\%) with bevacizumab, and 23 (20\%) with cetuximab. Details with respect to chemotherapy before RFA were as follows: a total of 66 patients (58\%) underwent RFA after one line, 36 (32\%) after two lines, and $11(10 \%)$ after 3 lines of prior systemic therapy. Most frequently applied last line pre-RFA regimens were FOLFOX (47\% [53/113] and FOLFIRI (37\% [42/113]). RFA was performed after PR in 63 patients (56\%); 21 patients (19\%) had SD, and 29 patients $(25 \%)$ had PD after the last line pre-RFA systemic therapy.

\section{RFA-related Efficacy and Complications}

Primary technical success was obtained in $93.6 \%$ (261 of 279) CLM. Secondary technical success was obtained in $100 \%$ (18 of 18 ) re-ablated CLM with residual enhanced tumour on follow-up CT $\leq 4$ weeks. Local RFA-site recurrence rates were $7.2 \%(20 / 279)$ on lesion basis, and $14.1 \%(16 / 113)$ on patient basis. Adverse events related to the RFA procedure were observed in $10.6 \%$ of patients $(12 / 113)$. Three patients $(2.6 \%)$ developed major complications (3x infected biloma requiring drainage and antibiotic therapy). The remaining 9 patients had one or more self-limiting minor complications (7 post ablation syndromes, 5 pleural effusions, 2 intrahepatic hematomas). There were no RFA-related deaths.

\section{Survival Outcome}

After a median follow-up from the date of RFA of 107 months (Interquartile range 83-127 months), the median OS for the entire cohort $(n=113)$ was 25.0 months (95\% confidence interval [95\% CI] 20.1-31.9), and the 5-year OS rate was $16.3 \%$ (95\% CI 9.9-23.9). Median RFS was 6.9 months (95\% CI 5.0-8.9) with a 5-year RFS rate of 7.1\% (95\% CI 3.1-12.5). OS was significantly higher for responders to systemic therapy before RFA (median 36.0 months; 29.1\% [95\% CI 18.3-42.2] at 5 years) than for non-responders (median 12.0 months; $0 \%$ at 5 years, $p<0.001$ ) (Figure 1). Similarly, RFS was different between responders 
(median 10.0 months; $12.7 \%$ [95\% CI 5.7-21.9] at 5 years) and non-responders (median 4.0 months, $0 \%$ at 5 years, $\mathrm{p}<0.001$ (Figure 1 ).

Table 1. Baseline data of the study cohort.

\begin{tabular}{|c|c|}
\hline Characteristic & Value \\
\hline Patients, n & 113 \\
\hline Male, n (\%) & $69(61)$ \\
\hline Female, $\mathrm{n}(\%)$ & $44(39)$ \\
\hline Median age (range), years & $70(36-88)$ \\
\hline Mean age $\pm S D$, years & $68.6 \pm 8.58$ \\
\hline \multicolumn{2}{|l|}{ Primary tumor } \\
\hline Colon, $\mathrm{n}(\%)$ & $89(79)$ \\
\hline Rectum, n (\%) & $24(21)$ \\
\hline \multicolumn{2}{|l|}{ Node status } \\
\hline Positive, $\mathrm{n}(\%)$ & $79(70)$ \\
\hline Negative, $\mathrm{n}(\%)$ & $34(30)$ \\
\hline \multicolumn{2}{|l|}{ Presentation of CLM } \\
\hline Synchronous, n (\%) & $24(21)$ \\
\hline Metachronous, $\mathrm{n}(\%)$ & $89(79)$ \\
\hline \multicolumn{2}{|l|}{ Number of CLM } \\
\hline Median number (range) & $2.5(1-5)$ \\
\hline Mean number \pm SD & $2.7 \pm 0.16$ \\
\hline Number of 1-3 CLM, n (\%) & $87(77)$ \\
\hline Number of 4-5 CLM, $\mathrm{n}(\%)$ & $26(23)$ \\
\hline \multicolumn{2}{|l|}{ Size of CLM, cm } \\
\hline Median maximum size (range) & $2.8(0.6-5.0)$ \\
\hline Mean maximum size $\pm \mathrm{SD}$ & $2.9 \pm 0.21$ \\
\hline Maximum size $\leq 3 \mathrm{~cm}, \mathrm{n}(\%)$ & $79(70)$ \\
\hline Maximum size $>3-5 \mathrm{~cm}, \mathrm{n}(\%)$ & $34(30)$ \\
\hline \multicolumn{2}{|l|}{ CEA levels before RFA, $\mathrm{ng} / \mathrm{mL}$} \\
\hline Median CEA level (range) & $\begin{array}{l}125.5 \\
(3-1.578)\end{array}$ \\
\hline Mean CEA level \pm SD & $81.3 \pm 15.6$ \\
\hline $\mathrm{CEA} \leq 100, \mathrm{n}(\%)$ & $71(63)$ \\
\hline CEA > 100, n (\%) & $42(37)$ \\
\hline \multicolumn{2}{|l|}{ Main cause of unresectability and/or inoperability } \\
\hline Expected liver remnant $\leq 30 \%, \mathrm{n}(\%)$ & $19(17)$ \\
\hline Proximity to critical structures, $\mathrm{n}(\%)$ & $25(22)$ \\
\hline Medical comorbidity, $\mathrm{n}(\%)$ & $52(46)$ \\
\hline Patients refusal, $\mathrm{n}(\%)$ & $17(15)$ \\
\hline \multicolumn{2}{|l|}{ Systemic therapies before RFA } \\
\hline Median number of lines (range) & $1.5(1-3)$ \\
\hline Mean number of lines $\pm \mathrm{SD}$ & $1.5 \pm 0.07$ \\
\hline$\geq 2$ lines of systemic therapy, $\mathrm{n}(\%)$ & $47(41)$ \\
\hline 5-Fluorouracile monotherapy, $\mathrm{n}(\%)$ & $43(38)$ \\
\hline Oxaliplatin-based combination therapy (FOLFOX), $\mathrm{n}(\%)$ & $75(66)$ \\
\hline Irinotecan-based combination therapy (FOLFIRI), $\mathrm{n}(\%)$ & $54(48)$ \\
\hline + Bevacizumab, $\mathrm{n}(\%)$ & $30(27)$ \\
\hline + Cetuximab, $\mathrm{n}(\%)$ & $8(7)$ \\
\hline \multicolumn{2}{|l|}{ Systemic therapies before and after RFA } \\
\hline Median number of lines (range) & $3(1-5)$ \\
\hline Mean number of lines $\pm S D$ & $3.1 \pm 0.1$ \\
\hline 5-Fluorouracile monotherapy, n (\%) & $76(67)$ \\
\hline Oxaliplatin-based combination therapy (FOLFOX), n (\%) & $106(94)$ \\
\hline Irinotecan-based combination therapy (FOLFIRI), n (\%) & $107(95)$ \\
\hline + Bevacizumab, $\mathrm{n}(\%)$ & $63(56)$ \\
\hline + Cetuximab, $\mathrm{n}(\%)$ & $23(20)$ \\
\hline \multicolumn{2}{|l|}{ Response to last line regimen before RFA } \\
\hline Partial remission, $\mathrm{n}(\%)$ & $63(56)$ \\
\hline Stable disease, $\mathrm{n}(\%)$ & $21(19)$ \\
\hline Progressive disease, $\mathrm{n}(\%)$ & $29(25)$ \\
\hline
\end{tabular}

CEA, carcinoembryonic antigen; CLM, colorectal liver metastases; RFA, radiofrequency ablation.

\section{Factors associated with Survival Outcome}

At the time of RFA treatment, five factors were independently associated with OS (Table 2): CEA level $(p=0.012)$, size of CLM $(p=0.022)$, number of CLM ( $p<0.001)$, number of lines of systemic therapy before RFA ( $p=0.001)$, and response to systemic therapy before RFA ( $p<0.001)$. Four factors were independently associated with RFS: size of CLM ( $\mathrm{p}=$ $0.001)$, number of CLM ( $p<0.004)$, number of lines of systemic therapy before RFA ( $p=0.017)$ and response to systemic therapy before RFA ( $p<0.001)$. Ranking of HRs revealed response to systemic therapy before RFA as the most important factor affecting OS (HR: 5.28, $\mathrm{p}<0.001$ ) and RFS (HR: 3.30; $\mathrm{p}<0.001$ ) (Table 2).

\section{Recurrence Pattern}

A total of 105 of the 113 (92.8\%) patients had recurrent disease (median time to recurrence: 6.1 months [range 1-24 months]). Most patients (83.8\% [88/105]) developed intrahepatic disease as a component of recurrence, and $65(61.9 \%)$ presented with liver-limited recurrence. Of the 41 patients $(39.0 \%)$ who experienced extrahepatic recurrence, 24 (58\%), $9(22 \%), 5(12 \%), 2(5 \%)$ and $1(2.5 \%)$ failed in the lung, regional lymph nodes, peritoneum, bone, and spleen, respectively, as the site of recurrence. There were 11 patients (10.5\%) who had recurrence only in the lung. Single organ site recurrences $(n=76)$ predominated over multiple organ site recurrences (n $=29$ ). The 65 liver-only recurrences accounted for the majority $(85.5 \%)$ of single site recurrences. Only 5 patients $(4.8 \%)$ had a local RFA-site recurrence as the sole site of recurrence.

\section{Recurrence Pattern and Survival Outcome}

After a median follow up from the date of recurrence of 99 months (Interquartile range 76-123 months), the median OS for the whole recurrence group $(\mathrm{n}=105)$ was 16.0 months $(95 \%$ CI 12.9-20.1) with a 5-year OS rate of $4.7 \%$ (95\% CI 1.3-9.9). Post-recurrence OS was significantly lower after early ( $\leq 6$ months) compared to later ( $>6$ months) recurrence (median 8.5 versus 24 months; $0 \%$ and $4.2 \%$ [95\% CI $2.6-18.9 \%$ ] at 5 years, $p<0.001$, Figure 2). With respect to the recurrence pattern, post-recurrence OS was as follows: a lung-only recurrence was associated with the best OS (median 39.9 months; 10.2\% [95\% CI $0.2-35.5 \%$ ] at 5 years); the next best OS was for patients with liver-only recurrence (median 19.1 months, $6.2 \%$ [ $95 \%$ CI $1.4-14.0 \%$ ] at 5 years), whereas those with multiple-site recurrence had the worst outcome (median 7.0 months, $0 \%$ at 5 years, $p<0.001$; Figure 2). 
Table 2. Univariate and multivariate analysis of factors associated with overall survival and recurrence-free survival after systemic therapy plus radiofrequency ablation of colorectal liver metastases.

\begin{tabular}{|c|c|c|c|c|c|c|c|c|c|c|c|}
\hline \multirow[t]{3}{*}{ Variables } & \multirow{3}{*}{$\begin{array}{l}\text { No. of } \\
\text { patients }\end{array}$} & \multicolumn{5}{|c|}{ Overall survival } & \multicolumn{5}{|c|}{ Recurrence-free survival } \\
\hline & & \multicolumn{3}{|c|}{ Univariate analysis } & \multicolumn{2}{|c|}{ Multivariate analysis } & \multicolumn{2}{|c|}{ Univariate analysis } & \multicolumn{3}{|c|}{ Multivariate analysis } \\
\hline & & $\begin{array}{l}\text { Median months } \\
(95 \% \text { CI })\end{array}$ & $\begin{array}{l}\text { 5-years, \% } \\
\text { (95\% CI) }\end{array}$ & p-value & HR $(95 \%$ CI $)$ & p-value & $\begin{array}{l}\text { Median months } \\
(95 \% \text { CI })\end{array}$ & $\begin{array}{l}\text { 5-years, \% } \\
(95 \% \mathrm{CI})\end{array}$ & p-value & HR $(95 \%$ CI) & p-value \\
\hline \multicolumn{12}{|l|}{$\begin{array}{l}\text { Patient and } \\
\text { tumor } \\
\text { characteristics }\end{array}$} \\
\hline Age & & & & 0.690 & & & & & 0.254 & & \\
\hline$\leq 60$ years & 21 & $21.9(15.9-38.0)$ & $15.7(3.4-34.7)$ & & & & $7.0(4.9-10.9)$ & $14.3(3.0-32.0)$ & & & \\
\hline$>60$ years & 92 & $25.0(20.9-31.0)$ & $16.5(9.5-25.0)$ & & & & $6.9(5.0-9.0)$ & $5.4(2.7-10.9)$ & & & \\
\hline Gender & & & & 0.643 & & & & & 0.730 & & \\
\hline Male & 69 & $25.0(18.0-31.1)$ & $15.2(7.4-25.1)$ & & & & $6.97(5.0-9.9)$ & $7.3(2.4-14.5)$ & & & \\
\hline Female & 44 & $25.0(18.1-36.0)$ & $18.2(8.3-30.8)$ & & & & $6.97(4.9-9.0)$ & $6.8(1.6-16.0)$ & & & \\
\hline Primary tumor & & & & 0.527 & & & & & 0.505 & & \\
\hline Colon & 89 & $24.0(18.0-31.1)$ & $15.1(8.2-23.6)$ & & & & $6.9(5.0-8.9)$ & $6.7(2.5-12.8)$ & & & \\
\hline Rectum & 24 & $30.0(22.0-36.9)$ & $20.8(7.3-38.9)$ & & & & $7.0(5.9-10.9)$ & $8.3(0.8-22.3)$ & & & \\
\hline Node status & & & & 0.924 & & & & & 0.967 & & \\
\hline Positive & 79 & $28.0(21.9-33.9)$ & $14.4(7.4-23.1)$ & & & & $6.9(5.0-9.1)$ & $7.6(2.8-14.4)$ & & & \\
\hline Negative & 34 & $20.0(16.0-31.9)$ & $21.5(9.1-37.4)$ & & & & $6.4(4.9-10.0)$ & $5.8(0.6-16.1)$ & & & \\
\hline CLM & & & & 0.967 & & & & & 0.056 & & \\
\hline Synchronous & 24 & $25.5(22.0-36.0)$ & $13.9(3.0-31.0)$ & & & & $9.5(6.0-11.9)$ & $4.2(0.7-15.6)$ & & & \\
\hline Metachronous & 89 & $25.0(18.0-33.0)$ & $17.0(9.7-25.7)$ & & & & $6.0(4.9-7.1)$ & $7.8(3.2-14.3)$ & & & \\
\hline $\begin{array}{l}\text { CEA level before } \\
\text { RFA }\end{array}$ & & & & $<0.001$ & $1.68(1.10-2.57)$ & 0.012 & & & 0.004 & & \\
\hline$\leq 100 \mathrm{ng} / \mathrm{ml}$ & 71 & $31.1(25.0-36.0)$ & $21.5(12.6-32.2)$ & & & & $8.9(6.9-10.0)$ & $9.8(4.1-17.8)$ & & & \\
\hline$>100 \mathrm{ng} / \mathrm{ml}$ & 42 & $15.5(11.1-25.0)$ & $7.5(1.5-17.6)$ & & & & $5.0(4.0-6.0)$ & $2.4(0.6-9.1)$ & & & \\
\hline $\begin{array}{l}\text { Maximum size of } \\
\text { CLM }\end{array}$ & & & & $<0.001$ & $1.78(1.09-2.90)$ & ) 0.022 & & & $<0.001$ & $2.18(1.36-3.48)$ & 0.001 \\
\hline$\leq 3 \mathrm{~cm}$ & 79 & $31.9(24.0-36.0)$ & $22.3(13.6-32.5)$ & & & & $9.0(6.9-10.0)$ & $10.1(4.5-17.7)$ & & & \\
\hline $3-5 \mathrm{~cm}$ & 34 & $14.5(10.0-22.0)$ & $2.94(2.0-11.1)$ & & & & $4.0(3.0-5.0)$ & $0(.-)$. & & & \\
\hline Number of CLM & & & & $<0.001$ & $2.68(1.62-4.46)$ & $<0.001$ & & & 0.012 & $1.99(1.25-3.17)$ & 0.004 \\
\hline $1-3$ & 87 & $29.0(23.0-35.0)$ & $20.17(12.3-29.3)$ & & & & $7.0(6.0-9.0)$ & $9.2(4.1-16.1)$ & & & \\
\hline $4-5$ & 26 & $13.5(10.0-26.9)$ & $0.00(-)$ & & & & $4.5(3.0-9.0)$ & $0(.-)$. & & & \\
\hline \multicolumn{12}{|l|}{$\begin{array}{l}\text { Systemic } \\
\text { therapy before } \\
\text { RFA }\end{array}$} \\
\hline $\begin{array}{l}\text { Lines of therapy } \\
\text { received }\end{array}$ & & & & 0.002 & $1.88(1.21-2.92)$ & 0.001 & & & 0.012 & $1.67(1.10-2.55)$ & 0.017 \\
\hline 1 & 66 & $31.9(26.9-36.0)$ & $18.2(9.7-28.7)$ & & & & $9.0(7.03-10.02)$ & $6.1(1.6-13.0)$ & & & \\
\hline$\geq 2$ & 47 & $13.0(10.0-17.9)$ & $13.7(5.3-25.2)$ & & & & $4.0(3.02-5.03)$ & $8.5(2.3-18.1)$ & & & \\
\hline $\begin{array}{l}\text { Oxaliplatin used } \\
\text { in last line }\end{array}$ & & & & 0.482 & & & & & 0.160 & & \\
\hline Yes & 53 & $24.0(18.0-35.0)$ & $20.1(10.4-31.9)$ & & & & $7.9(5.0-10.1)$ & $9.4(3.2-18.6)$ & & & \\
\hline No & 60 & $26.0(18.0-31.9)$ & $12.6(5.2-22.7)$ & & & & $6.0(5.0-8.0)$ & $5.0(1.0-11.9)$ & & & \\
\hline $\begin{array}{l}\text { Irinotecan used } \\
\text { in last line }\end{array}$ & & & & 0.673 & & & & & 0.140 & & \\
\hline Yes & 42 & $25.0(15.9-33.0)$ & $13.2(4.2-26.1)$ & & & & $5.5(4.9-7.0)$ & $7.1(1.4-16.7)$ & & & \\
\hline No & 71 & $25.0(18.0-34.0)$ & $17.7(9.7-27.5)$ & & & & $7.9(5.9-9.9)$ & $7.0(2.3-14.1)$ & & & \\
\hline $\begin{array}{l}\text { Bevacizumab } \\
\text { used in last line }\end{array}$ & & & & 0.425 & & & & & 0.920 & & \\
\hline Yes & 24 & $23.0(18.0-33.0)$ & $24.1(8.7-43.9)$ & & & & $6.0(4.9-7.0)$ & $12.5(2.6-28.3)$ & & & \\
\hline No & 89 & $26.0(18.0-33.0)$ & $14.4(7.8-22.6)$ & & & & $6.9(5.0-9.9)$ & $5.6(1.8-11.3)$ & & & \\
\hline $\begin{array}{l}\text { Cetuximab used } \\
\text { in last line }\end{array}$ & & & & 0.236 & & & & & 0.126 & & \\
\hline Yes & 8 & $34.5(22.0-38.5)$ & $25.0(3.1-58.4)$ & & & & $11.0(6.0-12.0)$ & $12.5(0.2-42.3)$ & & & \\
\hline No & 105 & $25.0(18.0-29.9)$ & $15.6(9.2-23.4)$ & & & & $7.0(5.0-8.0)$ & $6.7(2.7-12.2)$ & & & \\
\hline $\begin{array}{l}\text { Response to last } \\
\text { line regimen }\end{array}$ & & & & $<0.001$ & $5.28(3.18-8.77)$ & $<0.001$ & & & $<0.001$ & $3.30(2.09-5.20)$ & $<0.001$ \\
\hline $\begin{array}{l}\text { Partial } \\
\text { remission }\end{array}$ & 63 & $36.0(32.9-46.9)$ & $29.1(18.3-41.2)$ & & & & $10.0(9.0-12.0)$ & $12.7(5.7-22.0)$ & & & \\
\hline Stable disease & 21 & $21.9(15.9-25.0)$ & $0.00(.-)$. & & & & $5.9(4.9-7.0)$ & $0(.-)$. & & & \\
\hline $\begin{array}{l}\text { Progressive } \\
\text { disease }\end{array}$ & 29 & $9.03(8.0-10.9)$ & $0.00(.-)$. & & & & $3.0(2.9-4.0)$ & $0(.-)$. & & & \\
\hline
\end{tabular}




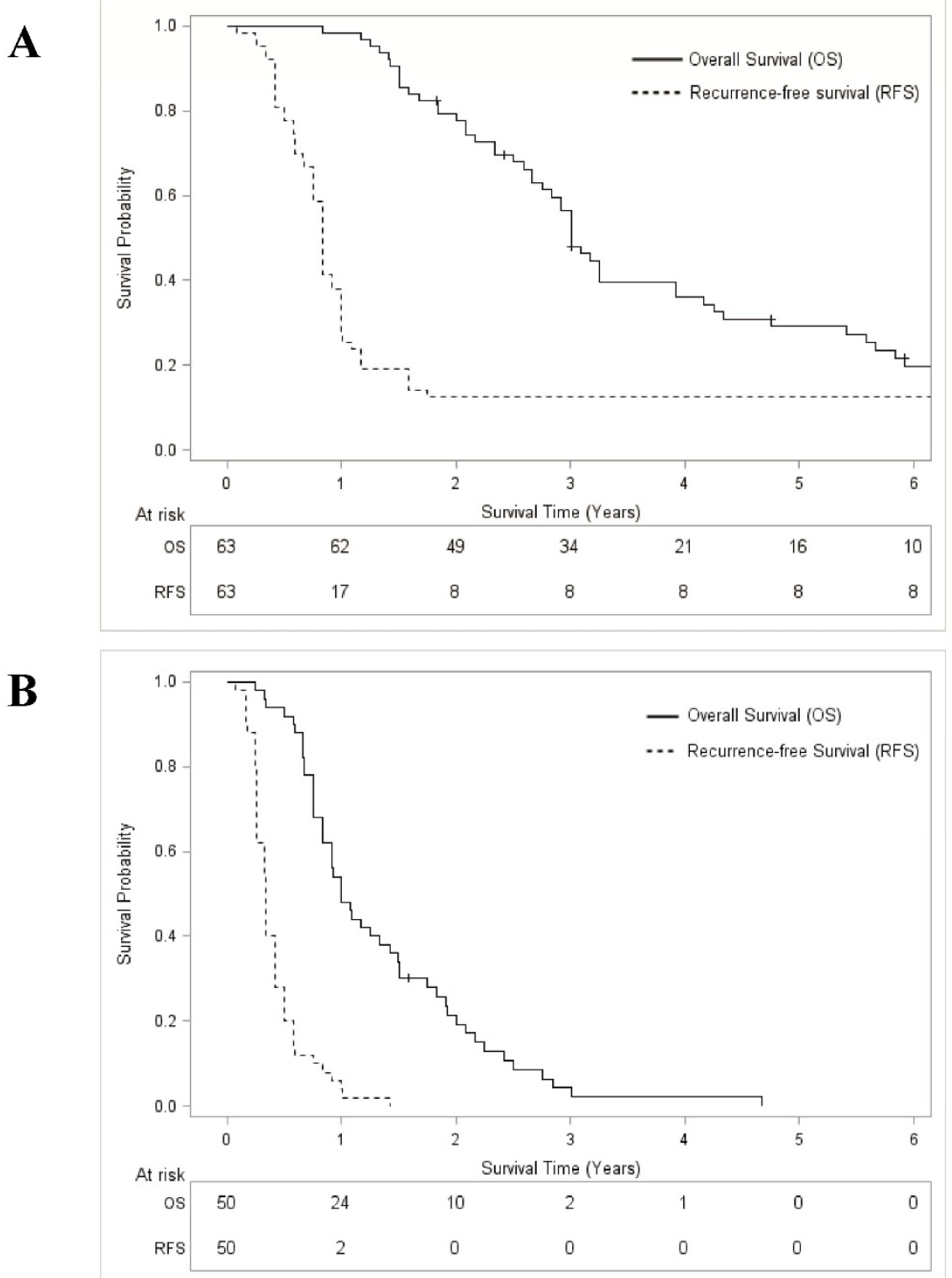

Figure 1. Overall survival and recurrence-free survival curves after systemic therapy plus radiofrequency ablation for colorectal liver metastases. A: Responders to systemic therapy; B: Non-responders to systemic therapy.

\section{Predictors of Recurrence Pattern}

At the time of RFA treatment, three parameters were independent predictors of early ( $\leq 6$ months) recurrence following RFA (Table 3): size of $>3 \mathrm{~cm}$ of CLM ( $p=0.005)$, number of $\geq 2$ lines of systemic therapy before RFA ( $p=0.027)$, and no response to systemic therapy before RFA $(p<0.001)$. Two parameters were independent predictors of multiple site recurrence: number of $>3$ RFA-treated CLM ( $p=$ 0.006 ) and no response to systemic therapy before RFA ( $p<0.001)$. Ranking of ORs identified no response to systemic therapy before RFA as the strongest predictor of early recurrence (OR 6.34, $\mathrm{p}<$ 0.001 ) and multiple site recurrence (OR 3.83, $p<0.001$ ) (Table 3). Associations between the timing and recurrence patterns are detailed in Table 4.

\section{Responders versus Non-responders to pre-RFA Systemic Therapy}

RFA was performed in 63 patients $(56 \%)$ during response (PR group) and in 50 patients $(44 \%)$ without response (non-PR group) after systemic therapy. Response was not related to the agents and/or regimens used, but both timing and patterns of recurrence differed significantly between the PR and non-PR group (Table 5). Response was associated with a 6 months longer RFS $(\mathrm{p}<0.001)$ and a higher rate of lung-only recurrence $(18.2 \%$ vs. $2.0 \%, \mathrm{p}=$ $0.007)$. By contrast, significantly higher rates of local RFA-site recurrence $(24.0 \%$ vs. $7.3 \%)$, multiple site recurrence $(40.0 \%$ vs. $16.4 \%)$, and intrahepatic recurrence $(90.0 \%$ vs. $68.2 \%)$ were observed in the non-PR group ( $p<0.05$, respectively, Table 5$)$. 

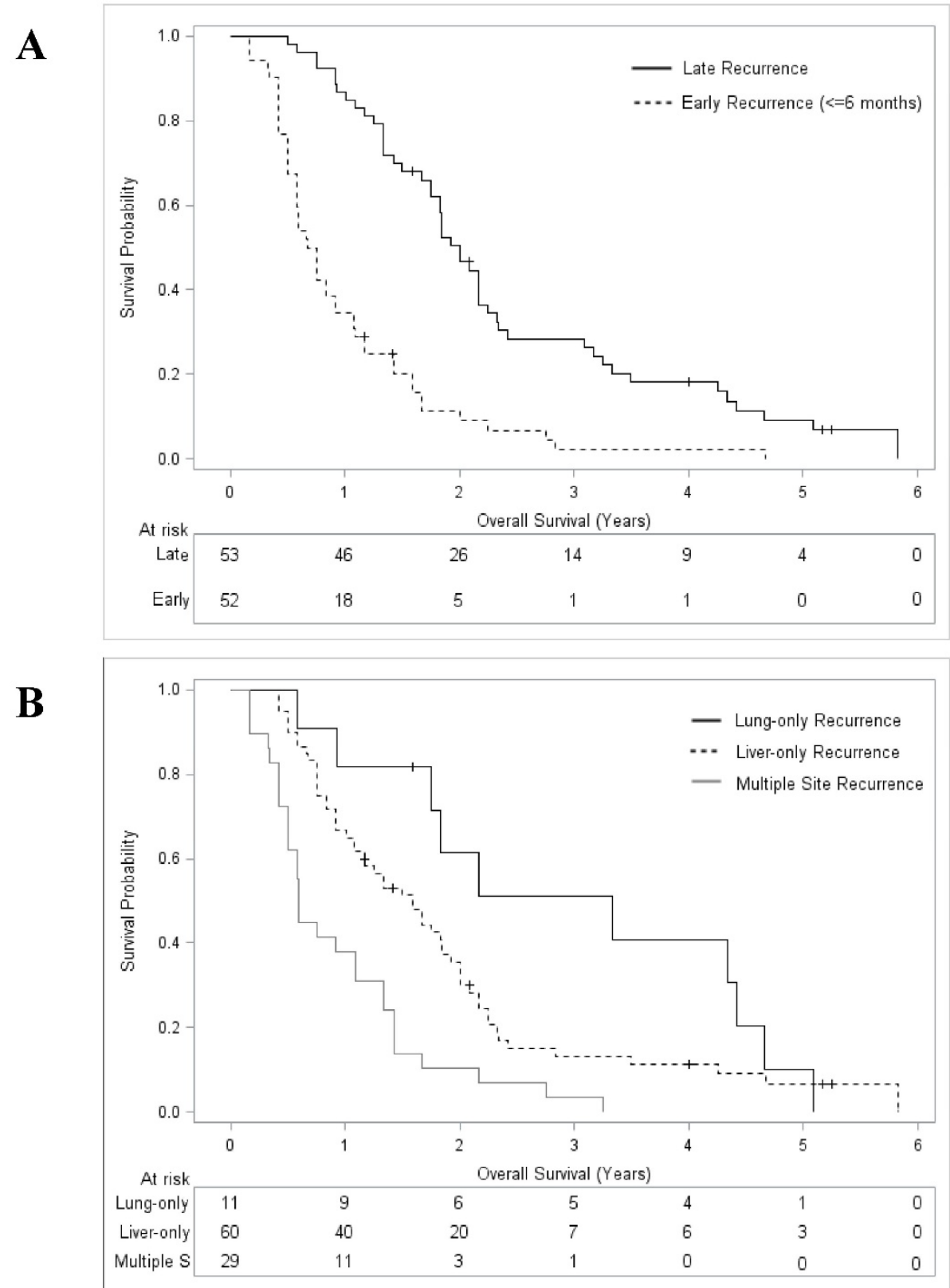

Figure 2. Overall survival curves from initial recurrence of disease after systemic therapy plus radiofrequency ablation for colorectal liver metastases. A: Stratified by recurrence timing; B: Stratified by recurrence sites.

Table 3. Univariate and multivariate analysis of factors associated with multiple $(\geq 2)$ site recurrence and early ( $\leq 6$ months) recurrence after systemic therapy plus radiofrequency ablation of colorectal liver metastases.

\begin{tabular}{|c|c|c|c|c|c|c|c|c|}
\hline \multirow[t]{3}{*}{ Variable } & \multirow{3}{*}{$\begin{array}{l}\text { No. of } \\
\text { patients }\end{array}$} & \multicolumn{3}{|c|}{ Multiple site recurrence } & \multicolumn{4}{|c|}{ Early recurrence } \\
\hline & & \multicolumn{2}{|c|}{ Univariate analysis } & Multivariate analysis & \multicolumn{2}{|c|}{ Univariate analysis } & \multicolumn{2}{|c|}{ Multivariate analysis } \\
\hline & & $\begin{array}{l}\text { Multiple site } \\
\text { recurrences n, (\%) }\end{array}$ & p-value & $\begin{array}{l}\text { OR } \\
(95 \% \text { CI })\end{array}$ & $\begin{array}{l}\text { Early recurrences } \\
\mathrm{n},(\%)\end{array}$ & p-value & $\begin{array}{l}\text { OR } \\
(95 \% \mathrm{CI})\end{array}$ & $\mathrm{p}$-value \\
\hline \multicolumn{9}{|c|}{ Patient and tumor characteristics } \\
\hline Age & & & 0.257 & & & 0.965 & & \\
\hline$\leq 60$ years & 18 & $7(38.9)$ & & & $9(50)$ & & & \\
\hline$>60$ years & 87 & $22(25.3)$ & & & $43(49.4)$ & & & \\
\hline Gender & & & 0.137 & & & 0.903 & & \\
\hline Male & 64 & $21(32.8)$ & & & $32(50)$ & & & \\
\hline Female & 41 & $8(19.5)$ & & & $20(48.8)$ & & & \\
\hline Primary tumor & & & 0.967 & & & 0.363 & & \\
\hline Colon & 83 & $23(27.7)$ & & & $43(51.8)$ & & & \\
\hline Rectum & 22 & $6(27.3)$ & & & $9(40.9)$ & & & \\
\hline
\end{tabular}




\begin{tabular}{|c|c|c|c|c|c|c|c|c|c|}
\hline \multirow[t]{3}{*}{ Variable } & \multirow{3}{*}{$\begin{array}{l}\text { No. of } \\
\text { patients }\end{array}$} & \multicolumn{4}{|c|}{ Multiple site recurrence } & \multicolumn{4}{|c|}{ Early recurrence } \\
\hline & & \multicolumn{2}{|c|}{ Univariate analysis } & \multicolumn{2}{|c|}{ Multivariate analysis } & \multicolumn{2}{|c|}{ Univariate analysis } & \multicolumn{2}{|c|}{ Multivariate analysis } \\
\hline & & $\begin{array}{l}\text { Multiple site } \\
\text { recurrences n, (\%) }\end{array}$ & p-value & $\begin{array}{l}\text { OR } \\
(95 \% \mathrm{CI})\end{array}$ & p-value & $\begin{array}{l}\text { Early recurrences } \\
\mathrm{n},(\%)\end{array}$ & p-value & $\begin{array}{l}\text { OR } \\
(95 \% \mathrm{CI})\end{array}$ & p-value \\
\hline Node status & & & 0.939 & & & & 0.625 & & \\
\hline Positive & 73 & $20(27.4)$ & & & & 35 (47.9) & & & \\
\hline Negative & 32 & $9(28.1)$ & & & & $17(53.1)$ & & & \\
\hline CLM & & & 0.215 & & & & 0.038 & & \\
\hline Synchronous & 23 & $4(17.4)$ & & & & $7(30.4)$ & & & \\
\hline Metachronous & 82 & $25(30.5)$ & & & & $45(54.8)$ & & & \\
\hline CEA level before RFA & & & 0.453 & & & & 0.002 & & \\
\hline$\leq 100 \mathrm{ng} / \mathrm{ml}$ & 64 & $16(25.0)$ & & & & $24(37.5)$ & & & \\
\hline$>100 \mathrm{ng} / \mathrm{ml}$ & 41 & $13(31.7)$ & & & & $28(68.3)$ & & & \\
\hline Maximum size of CLM & & & 0.009 & & & & $<0.001$ & $4.91(1.63-14.82)$ & 0.005 \\
\hline$\leq 3 \mathrm{~cm}$ & 71 & $14(19.7)$ & & & & $26(36.6)$ & & & \\
\hline $3-5 \mathrm{~cm}$ & 34 & $15(44.1)$ & & & & $26(76.5)$ & & & \\
\hline Number of CLM & & & 0.003 & $3.47(1.33-8.65)$ & 0.006 & & 0.337 & & \\
\hline $1-3$ & 79 & $16(20.2)$ & & & & $37(46.8)$ & & & \\
\hline $4-5$ & 26 & $13(50.0)$ & & & & $15(57.7)$ & & & \\
\hline \multicolumn{10}{|l|}{ Systemic therapy before RFA } \\
\hline Lines of therapy received & & & 0.346 & & & & $<0.001$ & $3.33(1.15-9.64)$ & 0.027 \\
\hline 1 & 62 & $15(24.2)$ & & & & $20(32.3)$ & & & \\
\hline$\geq 2$ & 43 & $14(32.6)$ & & & & $32(74.4)$ & & & \\
\hline Oxaliplatin used in last line & & & 0.745 & & & & 0.488 & & \\
\hline Yes & 48 & $14(29.2)$ & & & & $22(45.8)$ & & & \\
\hline No & 57 & $15(26.3)$ & & & & $30(52.6)$ & & & \\
\hline Irinotecan used in last line & & & 0.579 & & & & 0.058 & & \\
\hline Yes & 39 & $12(30.8)$ & & & & $24(61.5)$ & & & \\
\hline No & 66 & $17(25.7)$ & & & & $28(42.4)$ & & & \\
\hline Bevacizumab used in last line & & & 0.913 & & & & 0.435 & & \\
\hline Yes & 21 & $6(28.6)$ & & & & $12(57.1)$ & & & \\
\hline No & 84 & $23(27.4)$ & & & & $40(47.6)$ & & & \\
\hline Cetuximab used in last line & & & 0.091 & & & & 0.113 & & \\
\hline Yes & 7 & $0(0 \%)$ & & & & $1(14.3)$ & & & \\
\hline No & 98 & $29(29.6)$ & & & & $51(52.0)$ & & & \\
\hline Response to last line regimen & & & 0.002 & $3.83(1.48-10.85)$ & $0.001^{*}$ & & $<0.001^{*}$ & $6.34(2.29-17.56)$ & $<0.001$ \\
\hline Partial remission & 55 & $9(16.4)$ & & & & $13(23.6)$ & & & \\
\hline Stable disease & 21 & $6(28.5)$ & & & & $11(52.4)$ & & & \\
\hline Progressive disease & 29 & $14(48.3)$ & & & & $28(96.5)$ & & & \\
\hline
\end{tabular}

95\% CI, 95\% confidence interval; CEA, carcinoembryonic antigen; CLM, colorectal liver metastases; OR, odds ratio; RFA, radiofrequency ablation.

Table 4. Patterns and timing of disease recurrence after systemic therapy plus radiofrequency ablation of colorectal liver metastases.

\begin{tabular}{|c|c|c|c|c|}
\hline Variable & Any recurrence $(\mathrm{n}=105)$ & Early recurrence $\leq 6$ months $(n=52)$ & Late recurrence $>6$ months $(n=53)$ & p-value* \\
\hline \multicolumn{5}{|l|}{ Patterns of recurrence } \\
\hline Any local recurrence (LR), n (\%) & $16(15.2)$ & $8(15.4)$ & $8(15.1)$ & 0.967 \\
\hline LR, only $n,(\%)$ & $5(5.8)$ & $2(3.8)$ & $3(5.7)$ & 1.000 \\
\hline Any intrahepatic recurrence $n,(\%)$ & $88(83.8)$ & $48(92.3)$ & $40(75.5)$ & 0.019 \\
\hline Liver, only $\mathrm{n},(\%)$ & $65(61.9)$ & $30(57.7)$ & $30(56.6)$ & 0.910 \\
\hline Any extrahepatic recurrence, $\mathrm{n}(\%)$ & $41(39.0)$ & $21(40.4)$ & $20(37.7)$ & 0.781 \\
\hline Lung, only $n,(\%)$ & $11(10.5)$ & $1(1.9)$ & $10(18.9)$ & 0.005 \\
\hline \multicolumn{5}{|l|}{ Disseminated Recurrence } \\
\hline$\geq 2$ sites (disseminated), $\mathrm{n},(\%)$ & $29(27.6)$ & $19(36.5)$ & $10(18.9)$ & 0.043 \\
\hline
\end{tabular}

* Pairwise comparison between early and late recurrence.

Table 5. Comparison of systemic therapy and recurrence patterns between responders and non-responders to the last line systemic therapy before RFA treatment.

\begin{tabular}{llll}
\hline Variable & Responder (PR) & Non-Responder (SD,PD) & p-values \\
\hline $\begin{array}{l}\text { Number of patients (\%) } \\
\text { Systemic therapy received before RFA }\end{array}$ & $63(56)$ & $50(44)$ & $<0.001$ \\
$\quad$ & $1.2(1-3)$ & $1.8(1-3)$ & $<0.001$ \\
Median number of lines (range) & $14(22.2)$ & $33(66.0)$ & 0.864 \\
$\quad 2$ lines of chemotherapy, $\mathrm{n}(\%)$ & $30(47.6)$ & $23(46.0)$ & 0.579 \\
$\quad$ Oxaliplatin used in last line & $22(34.9)$ & $20(40.0)$ & \\
$\quad$ Irinotecan used in last line & &
\end{tabular}




\begin{tabular}{|c|c|c|c|}
\hline Bevacizumab used in last line & $12(19.1)$ & $12(24.0)$ & 0.523 \\
\hline Cetuximab used in last line & $5(7.9)$ & $3(6.0)$ & 1.000 \\
\hline \multicolumn{4}{|l|}{ Systemic therapy received before and after RFA } \\
\hline Median number of lines (range) & $2.9(1-5)$ & $3.2(2-5)$ & 0.011 \\
\hline Oxaliplatin & $57(90.5)$ & $49(98.0)$ & 0.131 \\
\hline Irinotecan & $57(90.5)$ & $50(100)$ & 0.033 \\
\hline Bevacizumab & $37(58.7)$ & $26(52.0)$ & 0.474 \\
\hline Cetuximab & $10(15.9)$ & $13(26.0)$ & 0.184 \\
\hline \multicolumn{4}{|l|}{ Timing of recurrence after RFA } \\
\hline Median time (range) to recurrence, months & $10.0(9.0-12.0)$ & $4.0(3.0-5.0)$ & $<0.001$ \\
\hline Time to recurrence $\leq 6$ months (early) & $13(23.6)$ & $39(78.0)$ & $<0.001$ \\
\hline Time to recurrence $>6$ months (late) & $42(76.4)$ & $11(22.0)$ & \\
\hline \multicolumn{4}{|l|}{ Patterns of recurrence after RFA } \\
\hline No recurrence & $8(12.8)$ & $0(0)$ & 0.009 \\
\hline Any local RFA-site recurrence (LR) & $4(7.3)$ & $12(24.0)$ & 0.028 \\
\hline LR, only & $2(3.2)$ & $3(6.0)$ & 0.867 \\
\hline Any intrahepatic recurrence & $43(68.2)$ & $45(90.0)$ & 0.039 \\
\hline Liver, only & $34(61.8)$ & $26(52.0)$ & 0.310 \\
\hline Any extrahepatic recurrence & $19(30.2)$ & $22(44.0)$ & 0.581 \\
\hline Lung, only & $10(18.2)$ & $1(2.0)$ & 0.007 \\
\hline \multicolumn{4}{|l|}{ Number of recurrence sites } \\
\hline$\geq 2$ sites (disseminated) & $9(16.4)$ & $20(40.0)$ & 0.007 \\
\hline
\end{tabular}

$\mathrm{PD}$, progressive disease; $\mathrm{PR}$, partial remission; RFA, radiofrequency ablation; $\mathrm{SD}$, stable disease.

\section{Discussion}

Traditionally, RFA has been used as single procedure for eradication of CLM from the liver to offer inoperable patients the chance of long-term OS. As a main problem following RFA involves the high risk of non-local disease recurrence, adjuvant systemic therapy may be an option to address occult microscopic disease, however, with uncertain responsiveness of macroscopic disease. The present study evaluates the prognostic importance of recurrence patterns in patients with liver-limited CLM rendered clinically disease-free after systemic therapy plus subsequent RFA treatment. The clinical importance of recognizing the prognostic impact of post-RFA recurrence patterns with known pre-RFA response of CLM is 2-fold: First, it reveals the relative benefits and limitations of RFA for disease control and patient outcome, and it provides direction for adjuvant strategies in the future.

With an overall recurrence rate of $92 \%$ and the predominance of non-local liver-limited recurrence $(57 \%)$, our data are close to those reported in previous RFA studies [5-9,21, 22]. To our knowledge, however, this study is the first to show that the timing and patterns of disease recurrence after RFA carry important prognostic significance. Early ( $\leq 6$ months) recurrence was associated with significantly lower post-recurrence OS than after late recurrence (8.5 versus 24 months), and recurrence in multiple sites was associated with significantly shorter median OS (7 months) when compared to liver-only recurrence (19 months) or lung-only recurrence (39 months). In addition, both early and multiple site recurrence were significantly associated. Therefore, in patients with complete ablatable CLM at presentation, the tumor biology can differ [23] and the associated course of recurrent disease and prognosis can vary. Despite complete tumor clearance from the liver by RFA, aggressive tumor biology may lead to an aggressive clinical course with poor patient outcome. This poor prognosis group of patients lacking realistic chance of long-term survival after RFA of liver-limited CLM have to be identified to avoid unnecessary RFA treatment.

Multivariate analysis outlined response to pre-RFA systemic therapy as the strongest independent predictor for OS (HR 5.28), RFS (HR 3.30), and prognostically poor early (OR 6.34) and/or multiple-site recurrence (OR 3.83) in our study population. Furthermore, only responders, but none of the RFA-treated non-responders, achieved 5-year survival without disease recurrence or 5-year OS. These effects were only related to response and not related to any particular agent and/or regimen used. Whether response simply identified the disease biology or whether effective systemic therapy modified the course of disease is impossible to prove in our study. We assume that drug efficacy against microscopic disease and indolent disease biology combined to influence for the better RFS and OS in our study population [24]. While the randomized phase II CLOCC trial documents an OS benefit from adding RFA upfront to systemic therapy [11], our study shows that response is prognostically important, rather than the use of systemic therapy itself. In addition to response, number $(\geq 2)$ of lines of pre-RFA systemic therapy, number $(\geq 4)$ and size $(\geq 3$ $\mathrm{cm}$ ) of CLM were also independent predictors of early and/or multiple site recurrence and poor outcome in 
our study cohort.

Our local (RFA-site) recurrence rate was 14.1\% per patient and $7.2 \%$ per lesion, which is comparable with rates reported in other RFA studies [4,21,22]. RFA-site recurrence occurred less frequently in responders to systemic therapy, likely because downsizing chemotherapy had eradicated micrometatases surrounding CLM [25]. More importantly, only 5 patients $(<5 \%)$ developed RFA-site recurrence as the sole site of recurrence, underscoring that non-local recurrence was the key determinant of prognosis in our series. However, outcome did not simply depend upon response to systemic therapy in our study cohort. Local ablation helped systemic agents to control disease at original sites, which was necessary, although not sufficient, to achieve long-term disease control. A long-term-result with a 5-year RFS rate of $12.7 \%$ seen in our RFA-treated responders would likely be unrealizable using systemic therapy alone, and supports a positive impact on long-term outcome from RFA-mediated eradication of responding CLM within this subgroup.

The predominant recurrence pattern was non-local liver-limited recurrence $(57 \%)$ in our study cohort. We and others noted a higher rate of liver-only recurrence after RFA (32-62.5\%) [14,21] compared to the $17-34 \%$ after resection of CLM [9,26,27], likely because hepatectomy removes parenchymal micrometastases who are at risk for future CLM $[22,28]$. This provides a rationale to investigate the role of adjuvant post-RFA liver-directed arterial therapy, such as hepatic arterial infusion, to target hepatic micrometastases [29]. In new studies evaluating adjuvant liver-directed arterial therapy, it will be important to assess clinical end points with benefit to patients. Our findings here suggest that RFA-treated responders to systemic therapy might constitute a proper study population to test whether improving hepatic RFS can lead to improved OS as well.

Our findings carry implications and provide direction for both clinical and research settings. First, RFA should not be used as single or upfront therapy for liver-limited CLM with uncertain responsiveness of disease. A selection process (test-of-time approach) by systemic therapy, with the objective to determine the long-term benefit, is necessary to identify those patients who may benefit from RFA and those who will not. Among patients that have progressive disease despite systemic therapy, subsequent RFA is not advocated, even in case of complete ablatable CLM. Second, the optimal timing of RFA is during maximum response and not when disease escapes systemic treatment. The optimal candidates for considering RFA are those with $\leq 3$ liver-limited CLM which were downsized after $1^{\text {st }}$ line chemotherapy to less than $3 \mathrm{~cm}$ in size. Third, following complete ablation of responding CLM, adjuvant liver-directed arterial therapy may be a considerable investigational approach.

The main limitation of this study is the retrospective nature of observing treated patients including a methodically non-avoidable selection bias. In addition, our observations were limited to a single-arm, single-institutional experience. However, we used a prospectively maintained data-base for information on recurrence. Therefore, the detection bias in our ascertainment of recurrence may be low. In addition, the magnitude of effects noted contributed to the significance of our findings. In spite of the limitations, we consider that our work may provide valuable information for the clinical practitioner and serve as a hypothesis-generating data-set for further clinical research.

\section{Conclusions}

Several notable findings of this study can be cited. First, local ablation efficacy is not sufficient, although necessary, to obtain long-term disease control following RFA for liver-limited CLM. Second, main challenge of disease control is the frequent occurrence of new metastases distant from RFA-treated CLM within the liver. Third, the timing and patterns of intrahepatic and/or extrahepatic disease recurrence carry important prognostic value. Fourth, effective pre-RFA systemic therapy does favourably affect the risk, timing, recurrence patterns and long-term survival. These findings carry implications for both clinical and research settings. First, a selection process by modern-agent therapy is essential before considering RFA of CLM, thereby allowing for the tailoring of RFA to maximize patient benefit, and following RFA of responding CLM, improving hepatic RFS will be the key to further OS benefit. Prospective multicenter trials are needed to consolidate our findings, ideally designed to evaluate the role of adjuvant post-RFA liver-directed arterial therapy as well.

\section{Competing Interests}

The authors have declared that no competing interest exists.

\section{References}

1. Mayer RJ, Venook AP, Schilsky RL. Progress against GI cancer during the American Society of Clinical Oncology`s first 50 years. J Clin Oncol 2014; 32: 1521-30.

2. Clark EM, Smith RR. Liver-directed therapies in metastatic colorectal cancer. J Gastrointest Oncol 2014; 5: 374-387.

3. Ahmed M, Solbiati L, Brace CL, Breen DJ, Callstrom MR, Charboneau JW, Chen MH, Choi BI, de Baere T, Dodd GD 3rd, Gervais DA, Gianfelice D, Gilliams AR, lee FT jr, Leen E, Lencioni R, Litrtrup PJ, Livraghi T, Lu DS, McGahan JP, Meloni MF, Nikolic B, Pereira PL, Liang P, Rhim H, Rose SC, 
Salem R, Sofocleus CT, Solomon SB, Soulen MC, Tanaka M, Vogl TJ, Wood BJ, Goldberg SN. Image-guided tumor ablation: standardization of terminology and reporting criteria - a 10-year update. Radiology 2014; 273: 241-260.

4. Tanis E, Nordlinger B, Mauer M, Sorbye H, van Coevorden F, Gruenberger T, Schlag PM, Punt CJ, Ledermann J, Ruers TJ. Local recurrence rates after radiofrequency ablation or resection of colorectal metastases. Analysis of the European Organization for Research and Treatment of Cancer \#40004 and \#40983. Eur J Cancer 2014; 50: 912-919.

5. Pathak S, Jones R, Tang JM, Pamar C, Malik H, Poston. Ablative therapies for colorectal liver metastases: a systematic review. Colorectal Disease 2014; 13: e252-e265.

6. Gilliams A, Goldberg N, Ahmed M, Bale R, Breen D, Callström M, Chen MH, Choi BI, de Baere T, Dupuy D, Gangi A, Gervais D, Helmberger T, Jung EM, Lee F, Lencioni R, Liang P, Livraghi T, Lu D, Meloni F, Pereira P, Piscaglia F, Rhim H, Salem R, Sofocleous C, Solomon SB, Soulen M, Tanaka M, Vogl T, Wood B, Solbiati L.Thermal ablation of colorectal liver metastases: a position paper by an international panel of ablation experts, the interventional oncology sans frontières meeting 2013. Eur Radiol 2015; May 22 [Epub ahead of print]

7. Solbiati L, Ahmed A, Cova L, Ierace T, Brioschi M, Goldberg SN. Small liver colorectal metastases treated with percutaneous radiofrequency ablation: local response rate and long-term survival with up to 10-year follow-up. Radiology 2012; 265: 958-968.

8. Shady W, Petre EN, Gonen M, Erinjeri JP, Brown KT, Covey AM, Alago W, Durack JC, Maybody M, Brody LA, Siegelbaum RH, D`Angelica MI, Jarnagin WR, Solomon SB, Kemeny NE, Sofocleous CT. Percutaneous radiofrequency ablation of colorectal cancer liver metastases: factors affecting outcomes - a 10-year experience at a single center. Radiology 2015 Aug 12 [Epub ahead of print]

9. Abdallah E, Vauthey JN, Ellis LM, Ellis V, Pollock R, Broglio KR, Hess K, Curley SA. Recurrence and outcomes following hepatic resection, radiofrequency ablation, and combined resection/ablation for colorectal liver metastases. Ann Surg 2004; 239: 818-827.

10. Ruers T, Punt C, van Coevorden F, Pierie JP, Borel Rinkes I, Ledermann JA, Poston G, Bechstein W, Lentz MA, Mauer M, van Cutsem E, Lutz MP, Nordlinger B. Radiofrequency ablation combined with systemic treatment versus systemic treatment alone in patients with non-resectable colorectal liver metastases: a randomized EORTC Intergroup phase II study (EORTC 40004). Ann Oncol 2012; 23: 2619-2626.

11. Ruers T, Punt C, van Coevorden F, Pierie JP, Borel Rinkes I, Ledermann JA, Poston G, Bechstein W, Lentz MA, Mauer M, van Cutsem E, Lutz MP, Nordlinger B. Radiofrequency ablation (RFA) combined with chemotherapy for unresectable colorectal liver metastases (CRC LM): long-term survival results of a randomized phase II study of the EORTCNCRI CCSG-ALM Intergroup 40004 (CLOCC). I Clin Oncol 2015; 33 ( suppl): abstr3501.

12. Ng KM, Chua TC, Morris DL. Resection and ablation for colorectal liver metastases. J Gastroint Dig Syst 2013; 3: 121.

13. Kingham TP, Tanoue $M$, Eaton A, Rocha FG, Do R, Allen $P$, DeMatteo RP, D`Angelica M, Fong Y, Janargin WR. Patterns of recurrence after ablation of colorectal cancer liver metastases. Ann Surg Oncol 2012; 19: 834-41

14. Govaert KM, van Kessel CS, Lolkema M, Ruers T, Borel Rinkes I. Does radiofrequency ablation add to chemotherapy for unresectable liver metastases? Curr Colorectal Cancer Rep 2012; 8: 130-137.

15. Cremolini C, Loupakis F, Antoniotti C, Lonardi S, Masi G, Salvatore L, Cortesi E, Tomasello G, Spadi R, Zaniboni A, Tonini G, Barone C, Vitello S, Longarini R, Bonetti A, DÀmico M Di Donato S, Granetto C, Boni L, Falcone A. Early tumor shrinkage and depth of response predict long-term outcome in metastatic colorectal cancer patients treated with first-line chemotherapy plus bevacizumab: results from phase III TRIBE trial by the Gruppo Oncologica del Nord Ovest. Ann Oncol 2015; 26: 1188-1195.

16. Lam VW, Spiro C, Laurence JM, Johnston E, Hollands MJ, Pleass HC, Richardson AJ. A systematic review of clinical response and survival outcomes of downsizing systemic chemotherapy and rescue liver surgery in patients with initially unresectable colorectal liver metastases. Ann Surg Oncol 2012;19:1292-1301

17. Knudsen AR, Kannerup AS, Mortensen FV, Nielson DT. Radiofrequency ablation of colorectal liver metastases downstaged by chemotherapy. Acta Radiol 2009; 50: 716-721.

18. Stang A, Oldhafer $\mathrm{KJ}$, Weilert $\mathrm{H}$, Keles $\mathrm{H}$, Donati $\mathrm{M}$. Selection criteria for radiofrequency ablation for colorectal liver metastases in the era of effective systemic therapy: a clinical score based proposal. BMC Cancer 2014; 14:500

19. Eisenhauer EA, Therasse $P$, Bogaerts I, Schwartz LH, Sargent D, Ford R, Dancey J, Arbuck S, Gwyther S, Mooney M, Rubinstein L, Shankar L, Dodd L, Kaplan R, Lacombe D, Verweij J. New response evaluation criteria in solid tumors: revised RECIST guideline (version 1.1). Eur I Cancer 2009; 45: 228-247.

20. Cardella JF, Kundu S, Miller DL, Milward SF, Sacks D. Society of Interventional Radiology clinical practice guidelines. J Vasc Interv Radiol 2009; 20(7 Suppl): S189-91.

21. Wong LS, Mangu PB, Choti MA, Crocenzi TS, Dodd GD 3rd, Dorfmann GS, Eng C, Fong Y, Giusti AF, Lu D, Marsland TA, Michelson R, Poston G, Schrag D, Seidenfeld J, Benson AB 3rd. American Society of Clinical Oncology 2009 clinical evidence review on radiofrequency ablation of hepatic metastases from colorectal cancer. J Clin Oncol 2010; 28: 493-508.
22. Stang A, Fischbach R, Teichmann W, Bokemeyer C, Braumann D. A systematic review on the clinical benefit and role of radiofrequency ablation a treatment of colorectal metastases. Eur J Cancer 2009; 45: 1748-1756.

23. Goasguen N, de Chaisemartin C, Brouquet A, Julie C, Prevost GP, Laurent-Puig P, Penna C. Evidence of heterogeneity within colorectal liver metastases for allelic losses, mRNA level expression and in vitro response to chemotherapeutic agents. Int J of Cancer 2010; 127: 1028-1037.

24. Panczyk M. Pharmacogenetics research on chemotherapy resistance in colorectal cancer over the last 20 years. World J Gastroenterol 2014; 20: 9775-9827.

25. Wakai T, Shirai Y, Sakata J, Valera VA, Korita PV, Akazawa K, Ajioka Y, Hatakeyama K. Appraisal of $1 \mathrm{~cm}$ hepatectomy margins for intrahepatic micrometastases in patients with colorectal carcinoma liver metastasis. Ann Surg Oncol 2008; 15: 2472-2481.

26. D'Angelica M, Kornprat $\mathrm{P}$, Gonen $\mathrm{M}$, DeMatteo RP, Fong $\mathrm{Y}$, Blumgart LH, Janargin WR. Effect on outcome of recurrence patterns after hepatectomy for colorectal metastases. Ann Surg Oncol 2011; 18: 1096-1103.

27. de Jong MC, Pulitano C, Ribero D, Strub J, Mentha G, Schulick RD, Choti MA, Adrighetti L, Capussotti L, Pawlik TM. Rates and patterns of recurrence following curative intent surgery for colorectal liver metastases: an international multi-institutional analysis of 1.669 patients. Ann Surg 2009; 250: 440-448.

28. Wakai T, Shirai Y, Sakata J, Kameyama H, Nogami H, Iiai T, Ajioka Y, Hatakeyama K. Histologic evaluation of intrahepatic micrometastases in patients treated with or without neoadjuvant chemotherapy for colorectal liver metastases. Int J Clin Exp Pathol 2012; 5: 308-314.

29. Kemeny NE, Janargin WR, Capanu M, Fong Y, Gewirtz AN, DeMatteo RP, D'Angelica MI. Randomized phase II trial of adjuvant hepatic arterial infusion to systemic therapy with or without bevacizumab in patients with resected hepatic metastases from colorectal cancer. J Clin Oncol 2011; 29: 884-889. 